\title{
PERIODIC RADIO EMISSION FROM THE HELIUM RICH STARS HD 37017 AND $\sigma$ ORI E
}

\section{FRANCESCO LEONE}

Osservatorio Astrofisico di Catania, Città Universitaria, I-95125 Catania Italy

GRAZIA UMANA

Istituto di Radio Astronomia del C.N.R., VLBI station, P.O. Box 169 Noto, Italy

ABSTRACT Drake et al. (1987) detected, for the first time, radio emission from magnetic Chemically Peculiar stars. They suggested that radio emission is consistent with gyrosynchrotron emission from continuously injected, mildly, relativistic particles trapped in a magnetic equatorial belt.

Leone (1991) showed that it is possible considering that the radio emission comes from magnetic poles and is due to the electron component of the wind flowing along the open field lines. As a consequence Leone (1991) put forward the hypothesis that such emission could be periodically variable.

In order to verify the surmise of a periodic radio emission, the magnetic Chemically Peculiar stars HD 37017 and $\sigma$ Ori E have been monitored with the VLA at $6 \mathrm{~cm}$.

By combining our data with those obtained by Drake et al. (1987) and Philips \& Lestrade (1988), we show that the radio emission from HD 37017 and $\sigma$ Ori $\mathrm{E}$ varies with the rotational period.

\section{INTRODUCTION}

Because of the presence of strong magnetic fields, non-thermal radio continuum emission from magnetic CP stars has been a subject of speculation for quite a long time but no detection has be claimed until Drake et al. (1987). By carrying out observations with the VLA at 2,6 and $20 \mathrm{~cm}$, Drake and co-workers detected radio emission of a few mJy in 5 out of 34 magnetic CP stars. Three of these stars being helium strong and two silicon stars. Thus it appears that among CP stars only the hotter ones are characterized by radio emission.

The interaction between the stellar wind and the magnetic field gives origin to envelopes whose shape is certainly non spherical. This is because, in principle, a stellar wind flows freely along the open field lines and stores matter between the closed lines (Shore 1987). Shore et al. (1987), studying ultraviolet CIV and SiIV line profiles and their dependence on the rotational phase for the star 
$\alpha S c l$, showed that the magnetosphere of a magnetic CP star co-rotates with the star itself. This result was confirmed by Shore \& Brown (1990) for some other helium strong stars. Since the magnetic dipole axis is inclined with respect to the rotational axis, the circumstellar envelope changes its projection onto the sky and a variable radio emission is expected.

For HD 37017 and $\sigma$ Ori E, Drake et al. (1987) reported a time resolved set of the radio flux measurements at 2,6 and $20 \mathrm{~cm}$. By phasing these measurements with the rotational period, Leone (1991) gave evidence of periodic variability. To verify the radio variability we performed observations of the stars HD 37017 and $\sigma$ Ori E with the VLA at $6 \mathrm{~cm}$.

\section{RADIO VARIABILITY}

For the helium strong stars HD 37017, assuming the ephemeris elements by Bohlender et al. (1987) we have phased our measurements with the ones by Drake et al. (1987) and Philips \& Lestrade (1988). Fig. 1 reports the radio variation as a function of the rotational phase.

Bohlender et al. (1987) reported the effective magnetic field curve, showing that $B_{e f f}$. presents a variation from zero to -.218 Tesla. Helium abundance is highest close to the magnetic field minimum phase, that is, it is highest at the magnetic poles (Pedersen 1979).

It is worthy to note that the $B_{\text {eff }}$. and helium variations can be described with a sinusoid like the radio variation. Since the radio curve presents its minimum close to the phase of the null effective magnetic field, helium abundance maxima correspond to radio maxima.

We have phased our data with the ephemeris elements Bohlender et al. (1987), referring to the helium strong star $\sigma$ Ori $\mathrm{E}$, with the measurements by Drake et al. (1987) and Philips \& Lestrade (1988). Fig. 2 reports the radio variations as a function of the rotational phase.

Bohlender et al. (1987) reported the $B_{\text {eff. }}$ curve, which shows a sinusoidal variation from +.281 to -.149 Tesla.

As for HD 37017, the helium concentrations are at the magnetic poles (Pedersen 1979). It is important to note that radio variation does not show a single wave behavior like the magnetic field, but a double-wave behavior like the helium abundance. Radio maxima correspond to magnetic extrema and radio minima correspond to the nulls of the effective magnetic field.

\section{ORIGIN OF THE RADIO EMISSION}

Because of the non-constant radio emission and flat spectral index of the radio flux, Drake and co-workers suggested that the emission is consistent with gyrosynchrotron emission from continuously injected, mildly relativistic, particles trapped inside a magnetic equatorial belt. The dimensions of the cavity are between two and ten stellar radii. However the above authors failed to identify the origin of such high energies, whereas Hunger et al. (1990) suggested that the relativistic electrons could be produced by the magnetic field line reconnection. Reconnection of magnetic field lines had already been proposed by Havnes \& Goertz (1984) as a possibility to prevent the magnetosphere from filling up to 


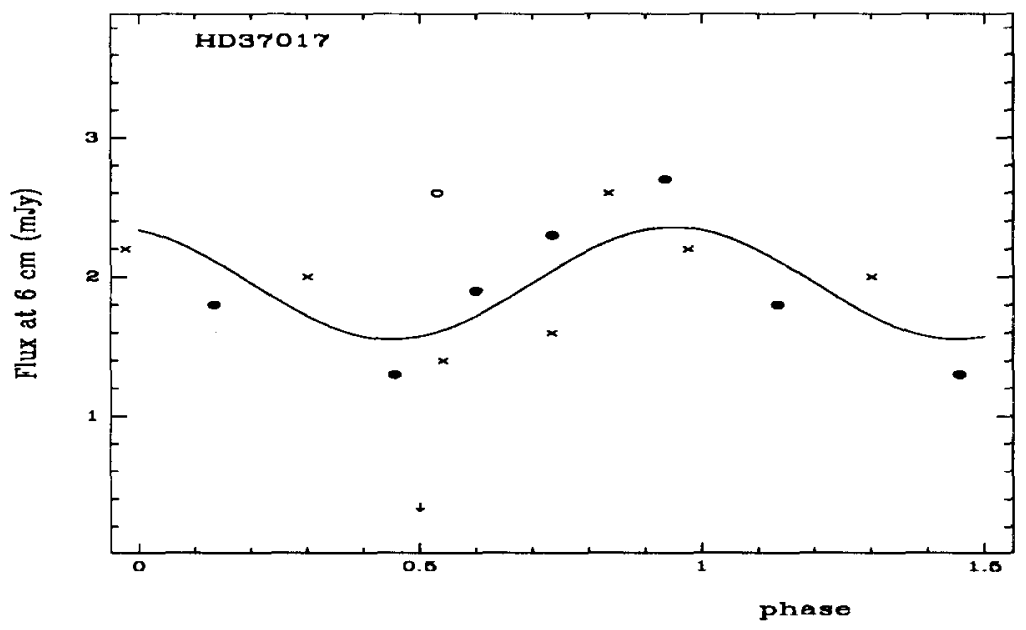

FIGURE I Radio light-curve at $6 \mathrm{~cm}$ of HD37017. $(x)$ stands for Drake et al. (1987) measures, (o) Philips \& Lestrade (1988) measure, (•) stands for our measures. The continuous line is sinusoidal fit. The arrow marks the phase of zero longitudinal magnetic field.

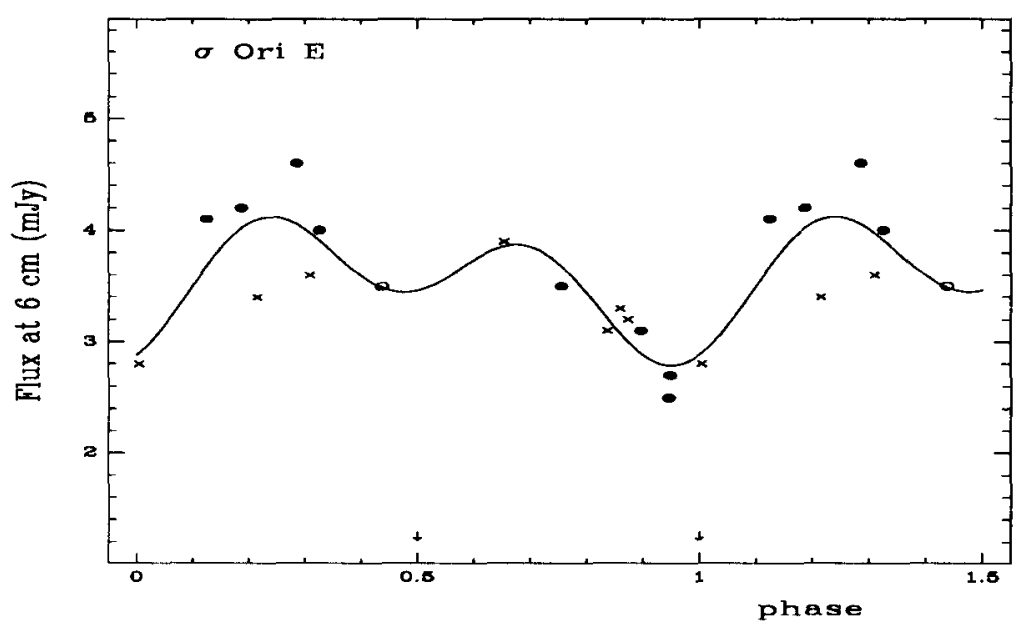

FIGURE II Radio light-curve at $6 \mathrm{~cm}$ of HD37017. ( $\mathrm{x}$ ) stands for Drake et al. (1987) measures, (o) Philips \& Lestrade (1988) measure, (•) stands for our measures. The continuous line is sinusoidal fit. The arrows mark the phases of zero longitudinal magnetic field. 
the Alfvén limit.

Andrè et al. (1988), drawing an analogy with $\rho$ Ophiuchi, discussed the radio emission of helium peculiar stars. Assuming the magnetosphere configuration by Havnes \& Goertz (1984) which, extending out to $10 R_{*}$, consists of a dense inner region where the electron number density is $\sim 10^{12} \mathrm{~cm}^{-3}$ and the temperature is $\sim 10^{4} \mathrm{~K}$, Andrè et al. (1988) did not share the explanation by Drake et al. (1987), because of the large free-free optical depth of the inner atmosphere (see also Leone 1991) and identified the emitting region with the middle magnetosphere.

Based on the magnetosphere dimension upper limit reported by Philips \& Lestrade (1988), Andrè et al. (1991) located the radio emission region in the inner magnetosphere suggesting that, in order to avoid plasma thermalization, the emitting electrons must be trapped in flux tubes.

Leone (1991) showed that the observed flat radio spectrum of magnetic CP stars could be due to gyroradiation from the electron component of the wind flowing along the open field lines of the magnetic field.

As to HD 37017 and $\sigma$ Ori E, evidences of stellar wind have been reported by Barker (1986). The response of the stellar wind to the presence of a dipolar magnetic field has been worked out by Shore (1987). He pointed out that in such conditions the stellar wind loses its central symmetry because of the reduced motion across the magnetic field lines, hence the wind of a magnetic star flows principally along the open field lines and matter is trapped between the closed field lines.

The coincidence of radio minima with the zero longitudinal magnetic field seems to exclude the gyrosynchrotron model by Drake et al. (1987). In fact, it has to be noted that radio emission from an equatorial belt, due to mildly relativistic electrons, should present its maximum at the zero longitudinal magnetic field and not, as it is observed, its minimum. This because force lines are orthogonal to the magnetic equatorial plane and an observer sees a relativistic electron radiating into a narrow cone about its instantaneous velocity.

In Leone's model the coincidence of radio maxima with magnetic extrema is due to the fact that gyroradiation is emitted principally along the magnetic field direction.

It is possible check the validity of Drake et al. (1987) and Leone (1991) models by observing HD 37017 at $1.3 \mathrm{~cm}$.

According to Leone the radio flux at $1.3 \mathrm{~cm}$ should be close to the detection threshold. In fact, electrons spiraling around the magnetic field lines with frequency $\nu_{H}=0.28 H \mathrm{GHz}$ ( $\mathrm{H}$ given in Tesla) emit radiation at the frequencies $s \nu_{H}$, where $s$ is the harmonic number, since for non relativistic electrons the first harmonic is the most important one, because of the HD 37017 dipolar magnetic configuration, no first harmonic is emitted at $1.3 \mathrm{~cm}$.

On the contrary, according to Drake et al. (1987) the radiation at $1.3 \mathrm{~cm}$ should be detectable. In fact, because of the assumed decrease of the magnetic field with the stellar distance cube, the volume emitting at $2 \mathrm{~cm}$ can be considered coincident with the volume emitting at $1.3 \mathrm{~cm}$. For example, if, as suggested, the ring around the magnetic equator emitting the radiation at $2 \mathrm{~cm}$ is 1.5 stellar radii from the star then the ring emitting at $1.3 \mathrm{~cm}$ is 1.4 radii from the star. Excluding abrupt discontinuities in the magnetospheric density, the flux values at 1.3 and $2 \mathrm{~cm}$ have to be close too. We observed HD 37017 at 


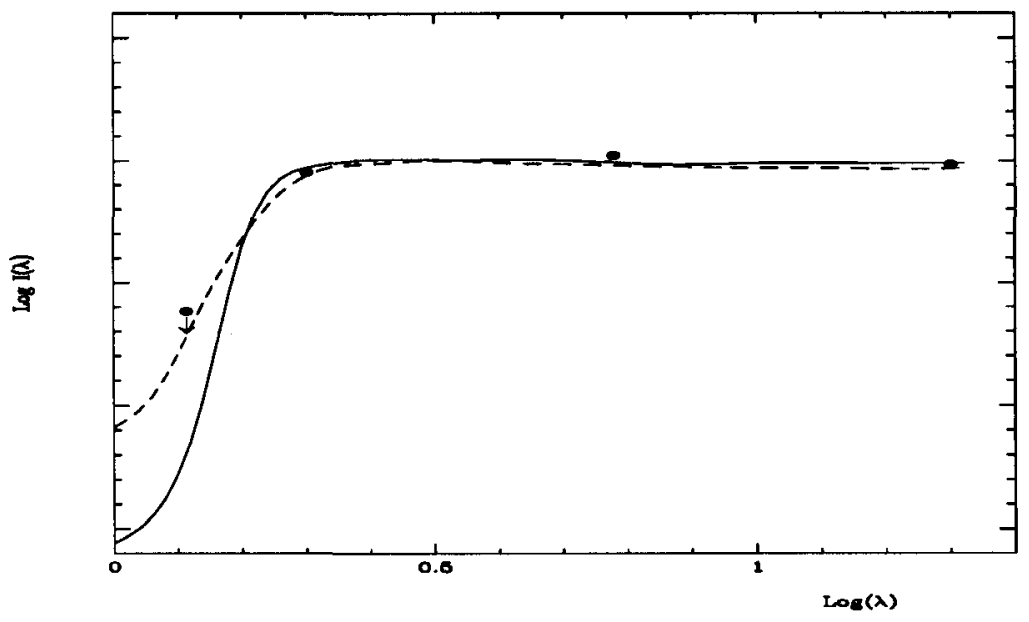

FIGURE III Circles represent in arbitrary units the radio flux for HD 37017 , at $1.3 \mathrm{~cm}$ it is reported the flux upper limit chosen three times the $\mathrm{rms}(=0.15 \mathrm{mJy})$ noise level on the map. Wavelengths are in $\mathrm{cm}$.. See text for the predicted radio emission spectrum superimposed to the 2,6 and $20 \mathrm{~cm}$ fluxes.

$1.3 \mathrm{~cm}$ and no radio source was found at the star positions. Fig 3 reports the radio flux upper limit, i.e. three times the $\mathrm{rms}$ noise level on the map ( $\mathrm{rms}=0.15$ $\mathrm{mJy}$ ), and the radio fluxes at 2,6 and $20 \mathrm{~cm}$ together with the expected radio spectrum due to gyroradiation emitted from the electron component of the wind channeled above the magnetic poles. The gyroradiation spectra has been calculated, according to Leone (1991), for an angle $\theta$ between the sight line and the dipole axis equal to $30^{\circ}$ (solid line) and $60^{\circ}$ (dashed line). The model spectrum has been superimposed on the observed radio flux at 2,6 and $20 \mathrm{~cm}$.

It is possible to conclude that the gyroradiation of the electron component of the wind can account for, not only the observed flat radio spectrum between 2 and $20 \mathrm{~cm}$ but, also the flux behavior at $1.3 \mathrm{~cm}$. It has to be noted that the calculated flux at $1.3 \mathrm{~cm}$ is larger at $\theta=60^{\circ}$ than at $30^{\circ}$. This is because there is no first harmonic at $1.3 \mathrm{~cm}$ and the relative flux is due to higher harmonics. According to Kundu (1965) the absorption coefficient, for low energies $\left(E \ll m c^{2}\right)$, for the highest harmonic increases with $\theta$.

\section{DISCUSSION AND CONCLUSION}

Our VLA observations confirm that the radio emission from the magnetic Chemically Peculiar stars HD 37017 and $\sigma$ Ori E is variable with the rotational period. As a direct consequence, we can affirm that the emitting volume is non spherical and co-rotates with the star. The phase relation between the radio and mag- 
netic field variations and the measured radio flux at $1.3 \mathrm{~cm}$ are in favor of the gyroradiation emission from the electron component of the wind flowing along the open field lines.

In order to explain peculiar abundances, Michaud (1970) proposed that some ions, driven by radiation pressure, should float in the stellar atmosphere, thus appearing overabundant, while other ions should sink, appearing underabundant. A model of helium behavior in the presence of a stellar wind and a magnetic field was presented by Vauclair et al. (1991). They simulated the magnetic field at the magnetic poles by vertical force lines and at the magnetic equator by horizontal force lines. Under these constrains, Vauclair et al. (1991) showed that helium is under abundant at the magnetic equator while it may accumulate at the polar regions. This helium distribution on the stellar surface was observed by Pedersen (1979) as to the star HD 37017 and $\sigma$ Ori E.

We can conclude that, if radio emission from magnetic CP stars is due to the electron component of the wind flowing along the open field lines above the magnetic poles, it is possible to consider the helium over-abundance at the magnetic poles and the radio emission as different aspects of the element diffusion, which is due to the radiation field and driven by the magnetic field.

\section{REFERENCES}

Andrè, P., Montmerle, T., Feiglson, E.D., Stine, P.C., Klein, K.L., 1988, ApJ 335,940

Andrè, P., Philips R.B., Lestrade, J.F., Klein, K.L., 1991, ApJ 376, 630

Barker, P.K., 1986, In: Hunger, K., Schönberber, D., Rao, N.K. (eds.) Proc.

IAU Col. 87, Hydrogen Deficient Stars and Related Objects, Reidel, Dordrecht, p. 284

Bohlender, D.A., Brown, D.N., Landstreet, J.D., Thompson, I.B., 1987, ApJ 323,325

Drake, S.A., Abbott, D.C., Bastian, et al. 1987, ApJ 322, 902

Havnes, O., Goertz, C.K., 1984, A\&A 138, 421

Hunger, K., Heber, U., Groote, D., 1990, In: Garmany C.D. (ed) Properties of Hot Luminous Stars. Astronomical Society of the Pacific Conference Series, Vol. 7, p.302

Kundu, M.R., 1965, Solar Radio Astronomy. Interscience Publishers. p.43

Leone, F., 1991, A\&A 252, 198

Michaud, G., 1970, ApJ 160, 641

Pedersen, H., 1979, A\&AS 35, 313

Philips, R.S., Lestrade, J-F., 1988, Nature 334, 329

Shore, S.N., 1987, AJ 94, 731

Shore, S.N., Brown, D.N., 1990, ApJ 365, 665

Shore, S.N., Brown, D.N., Sonneborn, G., 1987, AJ 94, 737

Vauclair, S., Dolez, N., Gough, D.O., 1991, A\&A 252, 618 